\title{
Figuraciones del silencio en la narrativa de la memoria: análisis desde el trauma ${ }^{1}$
}

\section{Silence's Figures in the Narrative of Memory: Analysis from Trauma}

\author{
Sandra Navarrete \\ Universidad de Santiago. Santiago, Chile. \\ sandra.navarrete.b@gmail.com
}

\section{Resumen}

En este artículo trabajamos dos novelas - Hija del silencio (2006) de Manuela Fingueret y La luz oscura (2013) de Nicolás Vidal- que focalizan la relación memorialística entre sujetos que han sufrido tortura y sus hijos. Nos interesa específicamente analizar los modos en que estas narrativas problematizan el trauma heredado por los hijos desde un tratamiento íntimo, psicológico e identitario, que destaca la necesidad de elaboración de la experiencia en el contexto familiar. Para ello, se trabaja especialmente la figura del "oyente" del testimonio y se configura una barrera entre "testigos internos" y "testigos externos" a la experiencia del horror. Nuestra hipótesis apunta a que en ambos textos la narración tensiona el espacio intersubjetivo del testimonio a partir de una estética del "silencio".

Palabras clave: trauma, memoria, silencio, testigo interno, testigo externo.

\begin{abstract}
The present study analizes two novels - Hija del silencio (2006) de Manuela Fingueret y La luz oscura (2013) de Nicolás Vidal- which focus on the relation between traumatized characters and their children, from the perspective of the latter. The novels that work on the trauma as something inherited and based on an intimate treatment of the character identity. Thus, the narration especially develops the figure of the "listener" testimony, and builds a barrier between "witness from inside" and "witness from outsinde". These narratives highlight the necessity of the elaboration of trauma in the familiar context. Our thesis is that this narratives build an intersubjectivity field where the bond between witness and listener defines an aesthetic of silence.
\end{abstract}

Keywords: Trauma, Memory, Silence, Witness from Inside, Witness from Outside.

1 Este artículo se enmarca en los proyectos Fondecyt Postdoctoral №3140072 y Fondecyt Regular No 1161551. 


\section{Los flujos inasibles de la memoria y el trauma}

Las reflexiones en torno a la memoria del Cono Sur constituyen un grosor crítico que ha conseguido ocupar un lugar importante dentro del campo del pensamiento latinoamericano. A partir de Maurice Halbwachs, la memoria ha dejado de asociarse exclusivamente a la capacidad individual de recordar hechos del pasado, para concebirse como una construcción social anclada en soportes colectivamente significativos. ${ }^{2}$ En esta misma línea, Elizabeth Jelin postula que la memoria es un ejercicio siempre dinámico y conflictivo en el cual diversos agentes se encargan de reinterpretar hechos del pasado, atendiendo o reaccionando ciertos hitos históricos que van marcando continuamente estos ejercicios. Importan aquí, por lo tanto, la revisión que los miembros de una comunidad realicen sobre su pasado y el sentido que se le otorgue al recuerdo, trabajo que estará determinado por un serie de factores sociales, políticos y culturales.

Con todos los matices y tipologías que han surgido para el término de memoria a partir de estas contribuciones, lo cierto es que se ha definido un nuevo y poderoso eje para abordar y comprender las formas en que el trauma es revisitado en países como Chile y Argentina. Dentro de este eje es posible identificar, claramente, la idea del deber de la memoria y la necesidad de revelar todas las vejaciones a los derechos humanos cometidas en períodos de dictadura, idea que en ambas naciones va aparejada con proyectos públicos que incluyen un conjunto de conmemoraciones, reparaciones simbólicas, museos y sitios de memoria, además de variadas acciones que tienen como trasfondo el carácter instrumental de estos actos. ${ }^{3}$

Nos preguntamos, entonces, ¿es la memoria siempre el resultado de una agencia, el producto de una actividad consciente sobre el pasado? Y si no es así, ¿qué aspectos dejamos fuera de foco con este concepto de memoria? Aun cuando creemos que la memoria funciona en gran medida gracias a este conglomerado de ejercicios oficiales, del mismo modo pensamos que es fundamental atender a aquellas manifestaciones soterradas o escurridizas que no consiguen posicionarse en el espacio público con un sentido tan evidente como los anteriormente mencionados, pero que de manera inevitable se manifiestan como un síntoma de algo aún no resuelto. En otras palabras, creemos que el recuerdo social es mucho más que un ejercicio de recuperación de hechos pasados, y que existen diversos flujos del recuerdo que no se deben a una acción concreta sobre estos. Nos referimos a aquellos terrenos incómodos, escasamente conscientes, pero al mismo tiempo ineludibles y que tienen que ver con lo que Régine Robin señala al hablar de un necesario giro en el enfoque memorialístico. Para la autora, es necesario catalogar "al mismo tiempo que los ritmos de la memoria, los modos de presencia del pasado en nosotros, la manera en que los pasados habitan el presente, y

2 Ver Los marcos sociales de la memoria, de Maurice Halbwachs.

3 Para Paul Ricoeur, es la justicia el principal elemento que le imprime utilidad a la memoria; de este modo, "al extraer de los recuerdos traumatizantes su valor ejemplar, transforma la memoria en proyecto; y es este mismo proyecto de justicia el que da al deber de memoria la forma del futuro y del imperativo" (119). 
no ya simplemente la manera en que el presente hace uso de los pasados" (239-40). "Los muertos se revelan", nos dice Robin, “vagan como espectros, fantasmas en los paisajes abandonados del presente, y no se dejan tan fácil olvidar o reclutar” (239). En este sentido, una manera específica de observar cómo el pasado se inmiscuye en el presente de los individuos y de las sociedades, sin el ejercicio consciente de actuar sobre dicho pasado, es el trauma. La violencia de la represión, las torturas y las desapariciones son la sombra que oscurece los procesos de duelo y desencadenan, de manera oscilante y compulsiva, una serie de reacciones fragmentarias, heterogéneas y cambiantes.

En atención a lo anterior, entenderemos el trauma como un núcleo semiótico que permite determinar los modos en que el pasado tortuoso se sigue haciendo espacio en la actualidad, no obstante el conjunto de consensos, olvidos y supresiones de la memoria, y más aún, a pesar de los mismos intentos de significar el pasado públicamente. Para ello hemos acotado un corpus de dos novelas, una chilena -La luz oscura (2013) de Nicolás Vidal- y otra argentina -Hija del silencio (2006) de Manuela Fingueret-, en las que analizaremos cómo se manifiesta el trauma en los sobrevivientes y sus consecuencias en la dificultosa relación con sus hijos. Estas novelas sitúan en primer plano la relación entre padres e hijos, reclamando los segundos la herencia de silencio que los primeros les han impreso en su propia identidad. De este modo, proponemos que la forma narrativa que adquiere el trauma se asienta en la necesaria intersubjetividad para la elaboración del mismo y, en la medida en que esta relación entre testigo y oyente no se produce, se instala un silencio avasallante y omnipresente en las nuevas generaciones. En otras palabras, postulamos que la tensión que genera la herencia de silencio de los padres hacia los hijos es el terreno en el cual ambas novelas posicionan el problema de la memoria. Estas narrativas se preguntan: ¿qué sucede cuando los sujetos traumatizados no elaboran su experiencia en un nivel púbico ni privado? ¿Cómo afecta este silenciamiento a las nuevas generaciones de la memoria? ¿Qué rol cumple el oyente del testimonio en la elaboración del trauma?

\section{Pactos íntimos de olvido y silencio: El pasado que no podemos escuchar}

El silencio es una manera de escribir los días del terror.

Y el silencio puede ser también la respuesta a toda imprecación de la memoria traumática

Escribir el horror. Literatura y campos de concentración, A. Cervera

Para aproximarnos a la presencia del silencio en el trauma propuesto en estas narrativas, delinearemos algunos conceptos fundamentales. En primer lugar, "trauma" es definido por Dominik La Capra como una experiencia específica y perturbadora, que es capaz de 
arrasar con la cotidianeidad que le da un sentido de integridad y articulación a la vida, lo que se produce al disociarse la cognición del afecto en el individuo y su relación con el entorno sociocultural, es decir, se desestabilizan las expectativas y la comprensión de los contextos, lo que genera una fractura irreconciliable. Quien padece un trauma, "siente, desconcertado, lo que no puede representar o representa anestesiado lo que no puede sentir" (La Capra 64) El pasado se repite una y otra vez en pesadillas, regresiones o "acting-out", que es cuando el sujeto revive la experiencia traumática, sin notar que está efectivamente en el presente. A partir de aquí, el "silencio" se puede entender como una consecuencia del trauma que imposibilita la representación del dolor, lo que desencadena un aura silente que conforma una barrera entre el "yo" y su entorno íntimo y social. ${ }^{4}$ Dentro del campo del psicoanálisis se destaca la línea relacional que propone que "la mente no es algo que nazca con el individuo, sino que se desarrolla en el entorno humano" (Rodríguez-Sutil 10), entendido este como una constelación de relaciones personales que posibilitan la constitución permanente del ser humano individual. En este sentido, la no comunicación del individuo con su entorno, posterior al evento traumático, evidencia un quiebre radical del ser individual al dañarse la "intersubjetividad" en sus relaciones con el mundo, lo que detiene la renovación y reactualización de su ser individual en el tiempo. En esta misma línea, entre las teorías psicoanalíticas sobre el trauma propiamente dicho, encontramos una tendencia que subraya la importancia de la interrelación entre dos sujetos para la superación de la experiencia. A partir de los trabajos fundadores de Cathy Caruth, Shoshana Felman y Dori Laub, se abre un nuevo campo para la relación entre el testigo y su otro/oyente. Según sus postulados, en el proceso de concientización de la memoria no asimilada por parte del sujeto del trauma es donde emerge el rol activo del oyente. Escribe Caruth: "It is the encounter and the coming together between the survivor and the listener, which makes possible something like a repossession of the act of witnessing. This joint responsibility is the source of the reemerging truth" ("An event without a witness" 85). Basándose en la propiedad inherente del trauma de ser siempre tardío (belatedness), esta línea de estudio explica que sin el oyente (listener) el trauma no puede ser recuperado. Es así como, después de un período de latencia, el sujeto de la experiencia comienza a elaborar el trauma sólo dentro de un marco de intersubjetividad (Caruth, Laub).

En el mismo sentido, Dori Laub explica que la existencia de un oyente para el trauma no es complementaria, sino que, ante todo, imprescindible para la elaboración de la experiencia. El testimonio del trauma incluye a su receptor, quien se constituye, por lo tanto, como una especie de pantalla en blanco en donde el evento traumático se escribe por primera vez: "El oyente del trauma se convierte en

4 Una posible salida es lo que La Capra denomina "working-through", aquel esfuerzo por volver a articular los afectos y las representaciones personales, esfuerzo que, a su vez, en un nivel público, permite la acción ética y sociopolítica de una determinada colectividad, con miras al futuro. El "working-through" que propone este autor no es tanto una cura como una especie de actitud compensatoria ante el trauma, que puede permitir que los individuos traumatizados se conviertan en agentes éticos (La Capra, cit. en Goldberg, s. p.). 
participante y co-propietario de la experiencia traumática: a través de su escucha, experimenta parcialmente el trauma por él mismo" (Laub 57).

En esta misma línea, Shoshana Felman reflexiona acerca de lo que significa testimoniar, ser testigos y sobrevivientes. Pensando la película Shoah de Claude Lanzmann como una obra de arte que pone en cuestión el acto mismo de testimoniar, la autora va definiendo dos espacios claves para entender la dinámica del testimonio, uno interno y otro externo al trauma. El primero corresponde a los campos de concentración y a las relaciones dentro del conjunto de víctimas y victimarios, propiamente dichos, y el segundo, a todo aquello que los rodea: judíos que no cayeron, espectadores alrededor del mundo e, incluso, las generaciones posteriores al Holocausto que estuvieron ligadas a los sobrevivientes y sus relatos.

En este sentido, en la frontera y tensión entre ambos espacios es donde se entreteje la problemática del testimonio, específicamente, las legitimaciones por la verdad histórica, la validez de la voz del sobreviviente y la pregunta por quién está habilitado, a fin de cuentas, para testificar: ¿aquellos testigos directos del horror, aquellos individuos que sufrieron tortura y habitaron campos de concentración, a quienes Felman denomina "testigos internos" (witness from inside), o también los "testigos externos" (witness from outside), aquellos que solo conocen el relato de la catástrofe? La lógica parece inclinarse por los primeros, pero acaso ¿es tan sencilla la respuesta? Para Felman, la respuesta no apunta al conocimiento de la verdad a través de la experiencia, sino que a la capacidad para brindar testimonio en una comunidad. Aquí es fundamental considerar que, generalmente, aquellos testigos directos viven esta verdad en forma de secreto. El horror es tan grande y tan avasallador que no solo es difícil o imposible de representar, sino que pesa sobre las conciencias de los sobrevivientes como una realidad que se debe ocultar. "Victims as well as executioners come to believe in their elected fate to join a tongue-tied cult of muteness, to be the destined bearers of the silence" (Felman 229). De este modo, si bien es el testigo interno quien ha experimentado la verdad del horror, es imposible para él representarlo y asumir el compromiso que implica testificar. "It is imposible to testify from inside because the inside has no voice [...] From within, the inside is unintelligible, it is not present to itself" (Felman 231). Para la autora, testimoniar es más que simplemente reportar un hecho que ha sido vivido, registrado o recordado. Significa asumir la responsabilidad por la verdad histórica y apelar a la empatía de una comunidad, por lo tanto, trasciende el alcance individual de la experiencia tras el testimonio. En este sentido, es necesario desplazar esta responsabilidad hacia los testigos externos, porque son solo ellos quienes pueden asumir el compromiso con el otro, implicado en el testimonio. Para ello, tienen que realizar un esfuerzo por acercarse al horror, por aproximarse a lo que el testigo interno vivió y que no puede representar. ${ }^{5}$

5 De este modo, trasladando o dislocando la responsabilidad de testificar en los espectadores del trauma, Felman explica que Claude Lanzmann construye un filme que se pregunta por el significado de ser testigo del Holocausto y demuestra que testimoniar no es solamente relatar la experiencia de horror, sino también -y en este caso, gracias al arte- asumir una posición ante el mismo. 
A partir de lo revisado, proponemos que los hijos de sobrevivientes se posicionan en la zona fronteriza del trauma que separa el horror de la vida cotidiana, desplazándose conflictivamente entre la experiencia de tortura (La luz oscura), el campo de concentración (Hija del silencio) y sus propias realidades espacio-temporales como hijos. De este modo, la tensión identitaria los sitúa oscilantemente como "testigos internos" y "testigos externos". En las dos novelas, observamos entonces cómo la memoria de los hijos no es más que un doloroso esfuerzo por romper el silencio de sus padres y así tratar de entender la experiencia de la catástrofe que es tan propia de sus padres como de ellos mismos.

\section{Trauma y memoria intergeneracional: el campo de las escuchas pendientes}

La narrativa latinoamericana reciente ha desarrollado un gran espacio para el cuestionamiento de la represión en dictadura, desde una mirada evaluadora que consigue vincular la violencia social actual con ese pasado de represión. Dentro de las ficciones de postdictadura, nos concentraremos en las chilenas y argentinas, en donde hay un grupo sólido y definido de narrativas que se posicionan desde la mirada de los hijos. Muchas de ellas son escritas efectivamente por hijos de detenidos desaparecidos, como se ha dado de manera importante en Argentina: las obras de Félix Bruzzonne, Laura Alcoba, Raquel Robles y Mariana Eva Pérez, entre otros. ${ }^{6}$ Por otro lado, también existen ficciones que focalizan la perspectiva de las nuevas generaciones a través de personajes principales que son "hijos de dictadura" y no necesariamente de detenidos desaparecidos, como observamos en la obra de Leonardo Sanhueza, Alejandro Zambra, Álvaro Bisama o Alejandra Costamagna. Lo que nos interesa de este corpus es cómo la voz de los hijos se ha hecho camino en la literatura contemporánea. Nos detendremos particularmente en los modos en que esta voz pone en primer plano la importancia del testimonio íntimo y familiar para el trabajo de la memoria colectiva, incluyendo el espacio privado en el tejido memorioso de una comunidad.

\subsection{Los recorridos laberínticos del trauma en La luz oscura, de Nicolás Vidal}

La novela de Nicolás Vidal nos cuenta la historia de Matías Gutiérrez, un joven abogado que descubre que su padre fue torturado en el Estadio Nacional, en los comienzos de la dictadura militar chilena. A partir del hallazgo de lo que parece ser un primer intento de testimonio de su padre, el protagonista comienza una investigación obsesiva para

6 Es importante destacar que, como en la literatura, así también en el arte fílmico encontramos una tendencia a dar a conocer la voz de los hijos de detenidos desaparecidos desde la estética de la indagación, como por ejemplo, en Los rubios (2003) de Albertina Carri o en la reciente El padre (2016) de Mariana Arruti. Es interesante que en ambas películas se tematice el silencio. 
comprender este suceso que le había sido ocultado. La narración se complejiza cuando van apareciendo fragmentos del relato del padre a partir de la lectura que Matías hace del mismo; de este modo, por un lado, el relato testimonial reclama atención, y por otro, el oyente se constituye como un elemento necesario: "El relato me obliga a volver hacia atrás, a mirar a mi padre, a tratar de entenderlo, a buscar explicaciones y respuestas que antes no había logrado encontrar. Pero no era fácil volver ahí. Mi estabilidad, en gran parte, descansaba en la idea de no hacerlo" (Vidal 18-9).

$\mathrm{Si}$ analizamos el inicio de la novela desde las expectativas lectoras, podemos conjeturar que se espera el descubrimiento de la verdad del padre condensada en el relato de su tortura, pero sin embargo, esta ficción opta por entregarnos el relato del proceso de búsqueda de esa verdad, de las preguntas que se va haciendo este hijo, de cómo va cambiando su vida a partir de este hito y qué niveles de perjuicio identitario y emocional alcanza Matías a medida que su investigación avanza. El protagonista de la ficción no será la víctima emblemática ni la historia principal será la de su tortura, sino que es la del hijo, este oyente dislocado que decide hacerse cargo del pasado secreto de su padre.

El armazón de la obra propone al trauma como núcleo de sentido que irá proyectando anillos semánticos en distintas direcciones, pero siempre remitiéndose a la escena de tortura del padre, la que por lo demás, ni el protagonista ni los lectores llegan nunca a conocer. Toda la primera parte de la ficción se concentra en el esfuerzo de Matías por recordar los primeros años de su infancia, etapa que coincide con el retorno de la familia desde España, país donde había llegado el padre exiliado. Durante este período, se observa la inadecuación, la violencia, pero sobre todo, el silencio continuo del padre con el entorno que lo rodeaba, mutismo que finalmente se extiende hasta su muerte: "Creo que fue esa elección, la del silencio, al que se mantuvo fiel por veintiocho años, la que lo pudrió. Tomó la forma de un cáncer a los huesos" (Vidal 65). El sujeto de la experiencia se representa como un individuo en constante insomnio, que dormía por el día y veía películas por las noches, alcohólico, violento, incapaz de transmitir emociones ni de comunicarse con sus seres más cercanos, delineando a través del silencio una frontera infranqueable con su familia. En este sentido, explica Marcelo Viñar: "Del retorno de quien ha vivido la experiencia traumática y de quien procura ser su interlocutor y testigo, jamás debemos subestimar las dificultades de este encuentro" (137). Es justamente aquí donde la narración construye la frontera que establece Felman entre los espacios interno y externo del trauma, que muestra finalmente cómo los "witness from inside" y "witness from outside" no pueden comunicarse. La focalización a cargo de Matías consigue otorgarle doble dimensionalidad a este campo minado que se configura alrededor del padre, ya que a través del recuerdo de la niñez refiere lo difícil de la convivencia, pero al mismo tiempo esta zona de comunicación tensionada se extiende hasta el presente de la enunciación, en donde aún persiste la pregunta por el silencio: “¿Cómo no me lo contaste?” (Vidal 118). Así, la historia del trauma del padre es tanto la del episodio de tortura como la de su 
sobrevivencia durante el resto de su vida, etapa en donde este hijo ahora se descubre como ignorante del sufrimiento de su progenitor.

Lo que encontramos es ante todo el deseo de escuchar esa experiencia de tortura, incluso después de la muerte del sujeto del trauma: "De alguna manera, tenía que obligar a mi padre a hablar; comprender a un fantasma, conversar con un muerto" (Vidal 66). ${ }^{7}$ Desde que Matías descubre el escrito testimonial de su padre, el trauma se convierte en un asunto propiamente identitario que define por completo a este personaje. La presencia silente de la memoria traumática va ocupando cada esfera de su vida y relegando todo aquello que constituía su diario vivir a un segundo plano: trabajo, amigos, pareja, hasta finalmente anularlos. Como explica Marcelo Viñar, los vacíos comunicativos en torno al trauma generan una serie de catástrofes en las personas involucradas: "una palabra enterrada en un padre puede transmitirse al psiquismo de un hijo" a través de lo que él denomina "fantasma". "El fantasma resulta del pasaje, de modo enigmático, del inconsciente de un padre al inconsciente de un niño", (140) el cual incorpora una serie de señales, afectos y signos que no logran encadenarse con un sentido claro y significativo para él sino que, por el contrario, crean un no-lugar, una no-existencia, un "fantasma". En suma, "el 'fantasma' indica los efectos sobre el descendiente de aquello que para el padre tuvo la cualidad de una herida, de una catástrofe psíquica" (140). Por lo tanto, el "fantasma" de Matías está en vida desde siempre, pero se detona a partir de la lectura del somero testimonio del padre, el que en vez de aclararle sus dudas, lo dejan sumido en la más dolorosa incertidumbre:

Vuelvo a sentirme débil, sediento, exhausto, el sueño no me deja pensar, nubla mis percepciones; el miedo, ahora solo queda el miedo. Veo soldados, algunos cabizbajos, avergonzados, y otros riendo orgullosos. Y veo también sus fusiles. Y sus culatas. Y sus bototos. Creí haber recobrado mis sentidos, pero me equivoqué. Todavía los veo: los he despertado (13).

El descubrimiento de la verdad oculta gatilla un proceso de apropiación del trauma por parte de Matías, proceso que termina por fundir corporalmente a ambos personajes: "Llevaba varios meses durmiendo pésimo, cada vez más consumido por la angustiosa desesperación del insomnio, para después durante el día, pasar a un estado de permanente tensión" (Vidal 124). Paulatinamente, el fantasma de la tortura se convierte en una ficción desgarradora que el hijo no puede evadir, ficción porque es menor la certeza y mayor lo imaginado respecto de la misma. El trauma persiste como fuerza opresora de la subjetividad del padre, en la primera generación, y luego permanece como un relato negado, en la segunda generación: "La tortura había estado dando vueltas como una presencia silente, observándome y escondiéndose” (Vidal 34). De este modo, la novela

7 Operación similar es la que realiza, por ejemplo, la novela de Leonardo Sanhueza La edad del perro (2014), en la cual el niño protagonista, hacia el final de la narración, se entera que su padre ha muerto, y sin embargo, ficcionaliza un encuentro post mortem con él, para realizarle las preguntas necesarias sobre su relación con la dictadura. 
se detiene en la necesidad del oyente de sanar las heridas, pero sobre todo discurre sobre qué sucede cuando definitivamente la interacción entre testigo y oyente no se produce, ahondando así en las consecuencias psicológicas de la no-elaboración del trauma, específicamente, en las relaciones filiales de los sujetos traumatizados. ${ }^{8}$

\subsection{Cautivas y mudas: Las mujeres en Hija del silencio, de Manuela Fingueret}

La novela de Manuela Fingueret nos relata las vidas de Tínkele y Rita, vidas entrelazadas por ser madre e hija, pero sobre todo, por la fuerte carga de horror que ambas padecieron en diferentes tiempos y espacios: Terezín/Auschwitz y la Escuela de Mecánica de la Armada (ESMA), respectivamente. El relato va urdiendo sus historias a través de un contrapunto libre que las va diferenciando por capítulo y por estilo tipográfico. De este modo, vamos conociendo sobre los modos en que Tínkele sobrevivió desde ese día de mayo de 1942, en que se la llevaron en un vagón de tren hacia Terezín, sin saber nunca más de sus padres. Por su parte, sobre Rita sabemos más de su vida previa a la ESMA que sobre su tortura y prisión, a pesar de que su testimonio es relatado desde la cárcel. El texto memorioso de Rita opta por volver hacia atrás, desde su infancia hasta la militancia propiamente dicha, deteniéndose detalladamente en aquellas mujeres que marcaron su vida, especialmente, en su madre y en el fuerte lazo que las une.

El testimonio en esta obra es doble: es tanto el de Tínkele como el de Rita, y se presentan, por un lado, a través de esa memoria intrusiva que es el relato biográfico en primera persona de la hija, y por otro lado, mediante un conjunto de capítulos que ordenan la experiencia de la catástrofe vivida por la madre, desde la perspectiva de un narrador omnisciente. Mientras el primer testimonio desarrolla una estética mixta que va mezclando la presentación y explicación de episodios de la vida de Rita con comentarios y reflexiones en un tono lírico, el segundo intenta organizar las vivencias de Tínkele, incluyendo indicaciones de tiempo y espacio precisas, que encabezan cada capítulo. La construcción secuencial y explicativa de la experiencia de la madre constituye todo aquello que Rita jamás supo, aquello que se ocultó tras el silencio pétreo de su progenitora: "Absorbí desde chica esa fragmentación, esa forma abrupta de callar. Me esforcé por encontrarle nuevos sentidos o cubrir los vacíos en cada una de estas charlas" (Fingueret 7).

La narración se encarga de representar estos dos testimonios como indisociables, pero al mismo tiempo, imposibles, en tanto el primero, el de la madre, nunca fue elaborado para la hija. No hay una revelación o un hallazgo de la historia de la madre, Rita jamás accede a su testimonio, y por lo tanto, nunca podrá tampoco elaborar el

8 El tópico de la filiación en relación con la memoria es un eje fundamental en la producción narrativa reciente, el que ha venido a renovar el género autobiográfico, tal como lo postula Sarah Roos al acotar la especificidad de los denominados "relatos de filiación". 
propio. Por ello, prefiere darle curso al relato de su infancia y adolescencia, y no a la tortura. De este modo, se privilegia resaltar la necesidad del vínculo dialógico para el testimonio dentro del contexto familiar: no transmitir la experiencia a las personas más cercanas deja huellas identitarias imposibles de borrar. El sufrimiento de la madre nunca abandona a la hija y se hace presente con más fuerza en la cárcel de la ESMA: "Es ella, otra vez, sosteniendo el vendaval de recuerdos con mate y leche. Ella, en este lugar, amarrada a mi piel, tatuada como un número" (Fingueret 12).

El "oyente" o listener, en la propuesta de Laub (1992), se entiende como un elemento necesario para que el trauma pueda ser elaborado por el testigo, y de este modo se convierte en copropietario y participante del trauma. En la novela de Fingueret observamos que, aun cuando el trauma no es elaborado y es el silencio el que impera en la relación madre-hija/testigo-oyente, Rita consigue igualmente acercarse a ese abismo que es el trauma y apropiarse de la experiencia de su madre. "El silencio contiene agujas que lo atraviesan todo" (18). Aquí, al igual que en la novela de Vidal, el trauma se hereda y no se supera, y el principal reclamo de los hijos es justamente el silencio que imperó en su infancia:

Cómo decirle lo que me faltó. Palabras que me explicasen lo que oculta más allá de esas cajas, de esos papeles arrugados, de esa estrella amarilla que descubrí por casualidad. Palabras que dieran sentido a los cuchicheos entrecortados. Palabras que pusieran nombre y contenido a Campo, tatuajes, exterminio. Y, sobre todo, palabras para su mirada oscura, volcada hacia adentro (140).

A diferencia de La luz oscura, en la novela de Fingueret la relación entre madre e hija durante la infancia no está mediada por la violencia o el desamparo. Si bien Rita añora haber podido compartir el testimonio de su madre, asume de manera comprensiva ese contacto especial con Tínkele: "Cuando la veía absorta, me sentaba a su lado en silencio. Ella viajaba hacia algún lugar inabordable y tarareaba cierta canción aprendida de su madre [...] Calladas, añorábamos sitios que alguna vez la habitaron. Un pacto secreto que -yo aún no lo sabía- nos uniría para siempre" (17). Insertas ambas en el silencio, el trauma es heredado, pero de alguna u otra manera, es igualmente comprendido, esa es la actitud que asume la hija: la disposición constante a ser "oyente" del testimonio desconocido de su madre; por eso, una vez adulta, asume la carga identitaria de este silencio. Como explica Daniela Goldfine en su análisis de la novela de Fingueret y Lenta biografía de Sergio Chejfec: "Es al final de sus vidas que los hijos advierten que siempre llevaron esa historia llena de recuerdos en sus cuerpos y en sus mentes -sus padres se la habían transmitido desde un lugar de mutismo" (93).

Con todo, el deseo de Rita en el presente sigue siendo que se rompa el pacto tácito de mutismo con Tínkele para así poder franquear el límite entre el espacio interno y el externo del trauma, del que habla Shoshana Felman. En este sentido, aunque en un comienzo se puede distinguir perfectamente a Tínkele como el "testigo interno" del horror y a Rita como el "testigo externo", a medida que avanza la narración esta 
distinción se va perdiendo: sus siluetas identitarias y sus cuerpos torturados se van homologando hasta casi convertirlos en uno solo:

Aún resuena en mi cabeza el chirrido de las gomas sobre el asfalto húmedo. Brazos que me levantaron en vilo como una pelota y tiraron adentro de un camión. El rebote contra el piso de metal, y un trayecto infinito en el que pensé en mi madre, en otro camión, en otro lugar, pero con la misma violencia. Ella en Minsk hacia Terezín; yo en Buenos Aires, hacia ¿dónde? Una casta de asesinos. Los nazis gaseaban, hambreaban, humillaban. Aquí insultan, torturan, violan. Casta de asesinos. Los escucho reír, jugar, beber... ¿Estoy allá o aquí? (65).

Distinguimos aquí un tratamiento narrativo que busca anular la frontera entre witness from inside y witness from outside mediante la configuración de ambos personajes -madre e hija-como víctimas de la misma catástrofe, es decir, al construir a Rita y Tínkele como sobrevivientes; de esta forma, además, la herencia del trauma resulta mucho más definida. Finalmente, se plantea la necesidad de que tanto la madre como la hija se constituyan a su vez como interlocutoras del testimonio de la otra, mediante una operación de intersubjetividad en la que Rita dialoga constantemente con su madre: ella es el hilo conductor de su memoria, el soporte textual y creativo de sus recuerdos.

\section{Estéticas del trauma y del silencio: apuntes de sus principales rasgos}

El trauma y el silencio son los puntos nodales construidos en estas dos novelas, a partir de los cuales se dirigen una serie de interrogantes sobre la narración del horror, la capacidad de testimoniar, el rol del oyente y la función que cumple la literatura en todo este proceso. Al igual que muchas de las novelas del amplio corpus de postdictadura, las obras que hemos analizado aquí se posicionan desde la escritura ficcional para ejercitar la memoria de un país y cuestionar distintos aspectos de este ejercicio. Lo que quisimos destacar a partir de esta aproximación es una variable específica que hemos advertido en las novelas de Vidal y Fingueret, y que hemos denominado "estética del trauma y del silencio", para poder acotar algunos aspectos característicos que nos han resultado sumamente interesantes de puntualizar.

En primer lugar, son narrativas en las que los hijos asumen la voz principal del relato para explicar, desde su perspectiva, el significado del trauma de sus padres en sus propias vidas. El silencio se define así como la consecuencia fundamental del hecho traumático en el sobreviviente y en las relaciones que posteriormente este establezca, y asume en este caso una envergadura definitoria, particularmente en las identidades de los hijos. De este modo, a pesar de que Matías y Rita son los protagonistas y narradores de la historia, su relato no se puede concebir sin la presencia arrolladora de sus progenitores. El que se privilegie el testimonio del oyente-hijo del sobreviviente, 
y no el del testigo directo, configura un giro de tuerca en el formato prototípico del relato testimonial -el cual suele concentrarse en el sujeto de la experiencia y en su dificultosa narración del trauma vivido-, y de esa forma da respuesta a la pregunta por lo que pasa cuando el episodio traumático jamás se elabora ni se supera en la primera generación, avanza hacia la segunda e indaga en los distintos modos de asumir dicho silencio por parte de los hijos. De esta forma, en el caso de la novela de Vidal, se puede apreciar lo que sucede cuando el padre opta por un silencio violento y tormentoso, que arrasa con la vida familiar de Matías, quien asume este trauma al borde del quiebre psicológico que lo lleva a un proceso de desvinculación con su madre, amigos, pareja, trabajo y todas las relaciones que lo rodean. En el caso de la novela de Fingueret, observamos un silencio cómplice asumido en formato de pacto entre madre e hija, en el cual se acepta cariñosamente la no-transmisión del trauma, en la medida en que la hija comprende la imposibilidad que amarra a su madre al silencio. No obstante lo anterior, una vez que la hija cae a su vez en prisión política, este trauma acallado sale a flote y la obliga a volver a su origen y revivir la experiencia abrumadora del silencio heredado.

En segundo lugar, en términos de la acción narrativa, se le otorga mayor importancia a la psique de los personajes que a sus acciones. En otras palabras, para la comprensión e interpretación de la novela, más que la sucesión de hechos relatados, importan las consecuencias que ellos han desencadenado en la psique de los personajes. Es así como la tortura, que es el hecho que debería erigirse como el principal núcleo de acción narrativa, es escasamente descrita en ambas novelas, mientras que sus consecuencias en la vida psíquica tanto de los padres como de los hijos ocupa la mayor densidad narrativa en ellas. La tortura es una especie de fantasma que acosa y acecha constantemente a dos generaciones, las cuales se constituyen en el vaivén ineludible que significa no poder alejarse del pasado y en la imposibilidad de comunicación con el presente.

En tercer lugar, son narraciones que consignan el rol fundamental del trauma en el seno del contexto familiar, en la vida íntima y privada, dejando que las memorias oficiales funcionen solo como un telón de fondo esbozado. En este línea de interpretación, si pensamos en el modo en que estas novelas insisten en la figura del oyente del testimonio, en su frustración frente al silencio de los padres, en sus ansias de indagación y en su solícita empatía, podemos pensar que tras este tamizado de intimidad se desarrolla subrepticiamente la idea del trauma social y de la necesidad de nuevos tipos de oyentes para el testimonio, unos que necesiten acceder al relato del horror para poder comprender sus propias identidades, unos que asuman ese llamado inconsciente y casi inaudible del trauma colectivo y que le den forma y voz. Finalmente, estas estéticas del trauma y del silencio permiten observar cuánto queda aún por reparar, cuánto silencio falta aún por elaborar, y así el testimonio de los hijos emerge como el relato de las escuchas pendientes, aquellas que aún esperan su espacio tanto privado como público. 


\section{Referencias}

Caruth, Cathy. "An event without a witness: truth, testimony and survival". Testimony. Crisis of Witnessing in Literature, Psychoanalisis and History. Nueva York, Routledge, 1992, pp.75-92.

---. "Introduction. The Wound and the Voice". Unclaimed Experience. Trauma, Narrative and History. Baltimore, The Johns Hopkins University Press, 1996, pp. 1-9. Felman, Shoshana. “The Return of the Voice: Claude Lanzmann's Shoah”. Testimony. Crisis of Witnessing in Literature, Psychoanalisis and History. Nueva York, Routledge, 1992, pp. 204-283.

Fingueret, Manuela. Hija del silencio. Buenos Aires, Booket, 2006.

Goldfine, Daniela. "La postmemoria del silencio: transmisión truncada y elipsis tangibles en la obra de Manuela Fingueret y Sergio Chejfec". Estudios Interdisciplinarios de América Latina y el Caribe, vol. 26, n 1, 2015, pp. 92-110.

Goldberg, Amos. “'Acting-out' and 'Working-through' Trauma”. Entrevista con Dominik La Capra. Shoah Resource Center, http://www.yadvashem.org/odot_pdf/ Microsoft\%20Word\%20-\%203646.pdf

La Capra, Dominik. Escribir la historia, escribir el trauma. Buenos Aires, Nueva Visión, 2005.

---. Historia en tránsito. Experiencia, identidad, teoría crítica. Buenos Aires, Fondo de Cultura Económica, 2006.

Laub, Dori. «Bearing Witness, or the Vicisitudes of Listening». Shoshana Felman y Dori Laub. Testimony. Crises of Witnessing in Literature, Psychoanalysis, and History. Nueva York, Routledge, 1992, pp. 57-74.

Robin, Régine. La memoria saturada. Buenos Aires, Waldhuter Editores, 2012.

Rodríguez-Sutil, C. "Epistemología del psicoanálisis relacional”. Clínica e Investigación Relacional, vol. 1, n 1, 2007, pp. 9-41, http://agorarelacional.es/Portals/0/ eJournalCeIR/V1N1/1_Rodriguez\%20Sutil_Epistemologia\%20del\%20Psicoanalisis\%20Relacional_CeIR_V1N1_2007.pdf

Vidal, Nicolás. La luz oscura. Santiago, Lom ediciones, 2013.

Viñar, Marcelo. «Violencia política y transmisión intergeneracional». Comp. Leticia Glocer, Los laberintos de la violencia. Buenos Aires, Asociación Psicoanalítica Argentina-APA, 2008, pp.133-152.

Enviado: 21 abril 2017

Aceptado: 31 enero 2018 